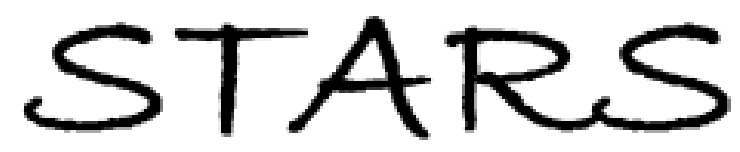

University of Central Florida

STARS

$1-1-1994$

\title{
Thermal Lens Coupled Magnetooptical Effect In A Ferrofluid
}

Tengda Du

University of Central Florida

Suihua Yuan

University of Central Florida

Weili Luo

University of Central Florida

Find similar works at: https://stars.library.ucf.edu/facultybib1990

University of Central Florida Libraries http://library.ucf.edu

This Article is brought to you for free and open access by the Faculty Bibliography at STARS. It has been accepted for inclusion in Faculty Bibliography 1990s by an authorized administrator of STARS. For more information, please contact STARS@ucf.edu.

\section{Recommended Citation}

Du, Tengda; Yuan, Suihua; and Luo, Weili, "Thermal Lens Coupled Magnetooptical Effect In A Ferrofluid" (1994). Faculty Bibliography 1990s. 1034.

https://stars.library.ucf.edu/facultybib1990/1034

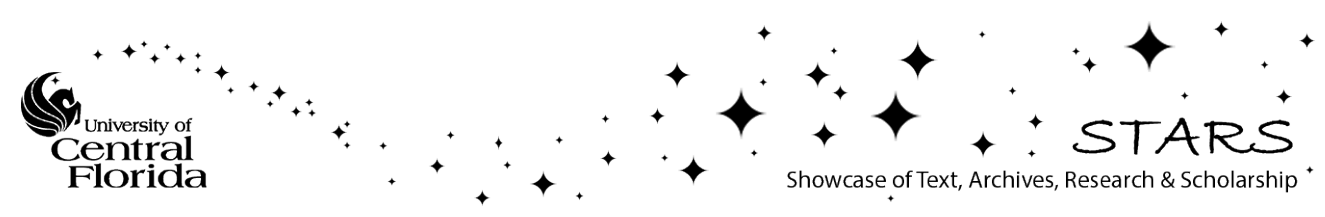




\section{Thermal lens coupled magneto-optical effect in a ferrofluid}

Cite as: Appl. Phys. Lett. 65, 1844 (1994); https://doi.org/10.1063/1.112861

Submitted: 03 June 1994 . Accepted: 28 July 1994 . Published Online: 04 June 1998

Tengda Du, Suihua Yuan, and Weili Luo

\section{ARTICLES YOU MAY BE INTERESTED IN}

Nonlinear optical effects in ferrofluids induced by temperature and concentration cross coupling

Applied Physics Letters 72, 272 (1998); https://doi.org/10.1063/1.120710

Long-Transient Effects in Lasers with Inserted Liquid Samples

Journal of Applied Physics 36, 3 (1965); https://doi.org/10.1063/1.1713919

Suppressing the thermal lens effect by magnetic-field-induced mass transfer and phase separation in a magnetic fluid

Applied Physics Letters 87, 021905 (2005); https://doi.org/10.1063/1.1996841

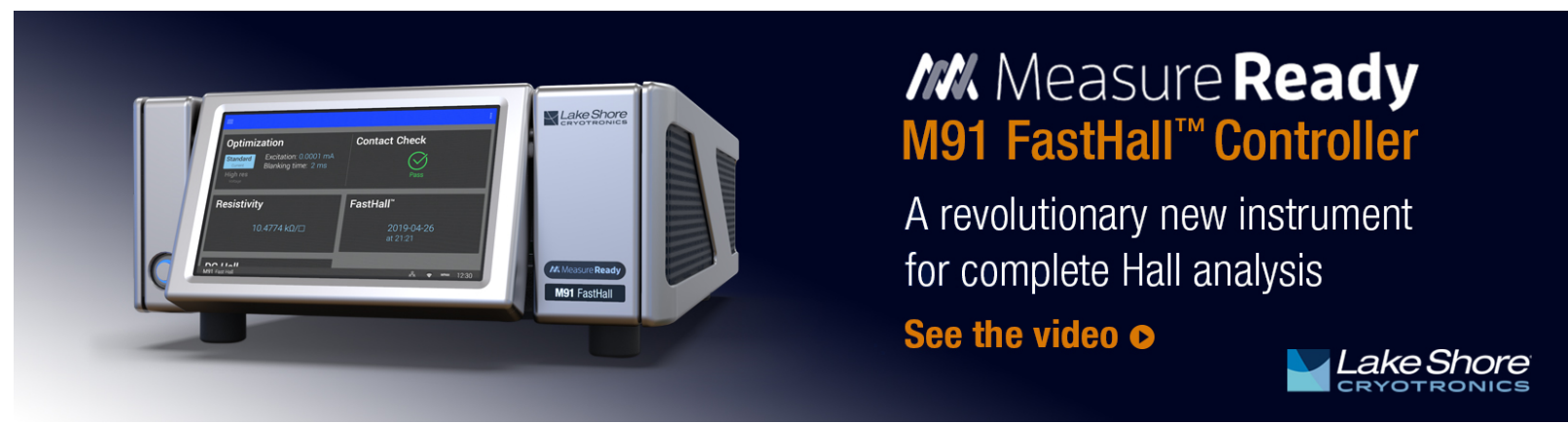




\title{
Thermal lens coupled magneto-optical effect in a ferrofluid
}

\author{
Tengda Du, Suihua Yuan, ${ }^{\text {a) }}$ and Weili Luo ${ }^{\text {b) }}$ \\ Department of Physics, University of Central Florida, Orlando, Florida 32816
}

(Received 3 June 1994; accepted for publication 28 July 1994)

\begin{abstract}
A magnetic-field-induced diffraction pattern was studied by a new technique: thermal lens coupled magneto-optical effect. In low fields, the diffraction angle has an almost quadratic dependence: $\theta \propto H^{2}$. This effect can be used as an all optical magnetic field sensor with a resolution of 0.5 Oe, with a potential for further improvement. The method can also lead to the measurement of the onset of agglomeration in the sample. (C) 1994 American Institute of Physics.
\end{abstract}

Although diffraction rings have been reported by several authors $^{1,2}$ in ferrofluids where the diffraction is attributed to the formation of micron-sized columns, light diffraction rings from single-domain magnetic particles in solution, to our knowledge, have never been observed before because the particle size is much smaller than the wavelength of the probing light. In this letter we will report a new, simple technique of light diffraction from a homogeneous ferrofluid and discuss the potential application of this method.

The sample is a ferrofluid consisting of magnetic particles (volume fraction 6\%) suspended in kerosene. Each particle has a diameter of $9 \mathrm{~nm}$ and is coated with a nonmagnetic surfactant layer of $2 \mathrm{~nm}$ in thickness. In Fig. 1 we show the main components of the experiment setup. A $10 \mathrm{~mW}$ $\mathrm{He}-\mathrm{Ne}$ laser beam with wavelength of $632.8 \mathrm{~nm}$ is focused normally on the sample slab. The sample cell has a dimension of $1 \mathrm{~cm} \times 1 \mathrm{~cm} \times 50$ microns. The focused beam waist is on the order of 10 microns. We found the confocal parameter $^{3}$ to be about 500 microns, thus the wave front in the medium can be considered as that of plane waves. The position of the lens needs to be adjusted carefully to have a clear diffraction image on the screen. The diffraction intensity profile is captured by a CCD camera and patterns are digitized by a computer. The magnetic field is applied normally to the sample and is in parallel with the laser beam.

In Fig. 2 we show the effect of a focused beam on a ferrofluid in comparison with that of an unfocused beam. Figure 2(a) shows the forward scattering from the sample on the screen in zero magnetic field and when the incident beam is not focused. Only one beam spot is visible. Figure 2(b) is the result of diffraction in zero field when the incident beam is focused by a lens as illustrated in Fig. 1. When the beam center is shifted to a new location, new diffraction rings reappear in a few seconds. These patterns have been observed in other materials as mentioned in Ref. 4 and are attributed to the thermal lens effect. The Gaussian profile of the focused beam leads to a temperature distribution in which the center of the beam has the highest temperature: ${ }^{4}$

$$
T(r)=T_{0}+\frac{W_{0}^{2} A}{4 K} \int_{0}^{r} d r^{\prime}\left(e^{-2 r^{\prime 2} / W_{0}^{2}}-1\right) / r^{\prime},
$$

where $T_{0}$ is the temperature at the beam axis, $K$ is the ther-

\footnotetext{
${ }^{a}$ On leave from Xian Jiaotong University, China.

${ }^{b)}$ To whom correspondence should be addressed.
}

mal conductivity of the fluid, and $A$ is independent of $r$ but is instead a function of the laser power and the absorption of the fluid. Gordon et al. also proved that thermal convection of the fluid had a trivial contribution to the temperature distribution. The density of the medium, in response to the temperature gradient, redistributes in a way such that it is the lowest at the center of the beam. The system with the new density distribution behaves like a concave lens because of the refractive index distribution. This is also called "thermal lens effect." The change in the local index of refraction is proportional to the local temperature change: ${ }^{5} \delta n(r)_{\text {th }}$ $=(\partial n / \partial T) \Delta T(r)$.

Applying a magnetic field has two effects on the diffraction pattern. (1) First, the diameters of the diffraction rings increase with increasing field and (2) at $H=12 \mathrm{Oe}$, a new ring appears. Figure 2(c) shows the diffraction rings in the field of 15 Oe. When the thermal-lens effect is absent, i.e., when the incident beam is not focused, no diffraction ring is observed for fields up to 180 Oe as shown in Fig. 2(d).

When a magnetic field is applied, we noticed that the field introduces an extra change in the refractive index beside the one arisen from thermal effect: ${ }^{6}$

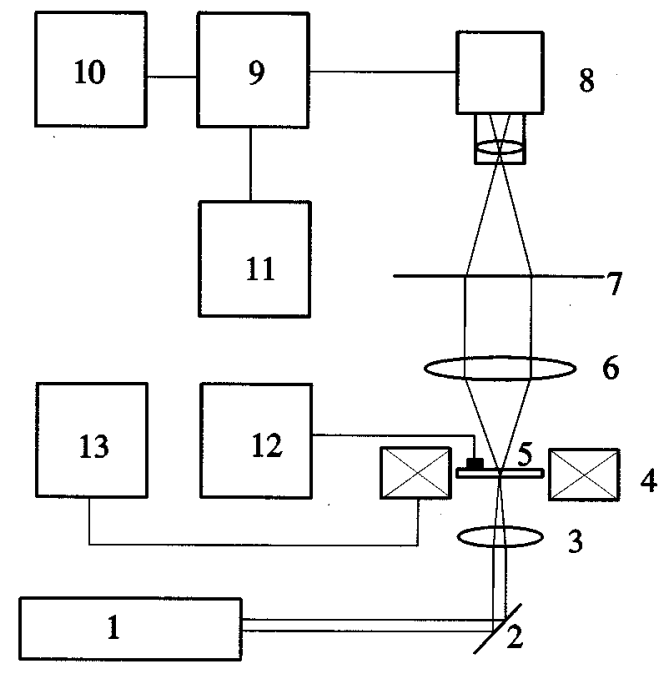

FIG. 1. The set up for light scattering experiment. 1-10 mw He-Ne laser. 2-mirror. 3-lens for focusing the incident beam. 4-coil for producing magnetic fields. 5-sample cell. 6-lens. 7-screen. 8-CCD camera. 9 -video recorder. 10 - computer. 11 - monitor. 12 - Gauss meter. 13 power supply. 


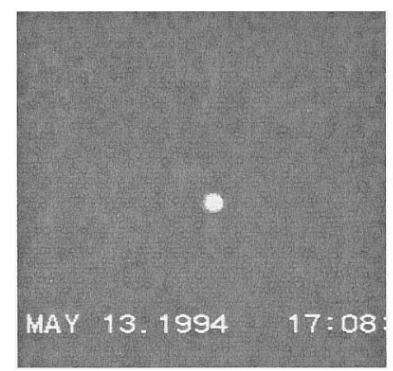

(a)

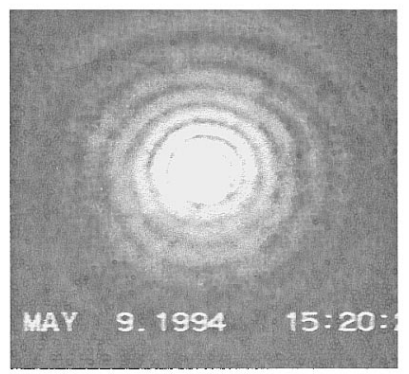

(c)

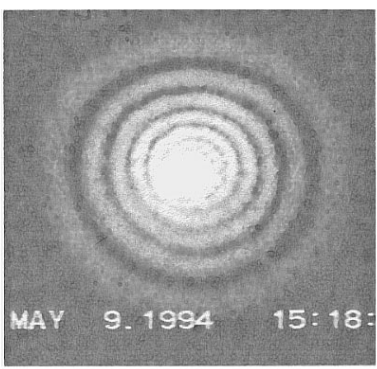

(b)

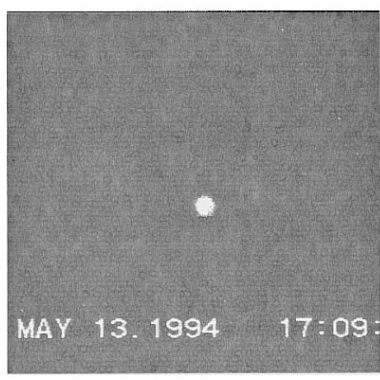

(d)
FIG. 2. Steady state diffraction patterns: (a) When lens 3 in Fig. 1 is absent, the incident beam passes through the ferrofluid sample without diffraction, leaving a bright spot on the center of the screen. (b) The diffraction pattern observed when the laser beam is focused on the sample in zero applied field-the thermal lens effect. (c) Diffraction patterns with thermal lens effect in a field of 15 Oe. (d) When thermal lens effect is absent, applied magnetic field does not produce diffraction rings for field up to 180 Oe. The spot shown here is in a field of 40 Oe.

$$
\delta n(H, r, z)_{H}=\frac{1}{2} n_{0} f\left[\alpha_{\perp}+F(H, r, z) \Delta \alpha\right],
$$

where $n_{0}$ is the refractive index of kerosene, $f$ is the volume fraction of the magnetite particles in the ferrofluid, $\alpha_{\perp}$ and $\alpha_{\|}\left(\Delta \alpha=\alpha_{\|}-\alpha_{\perp}\right)$ are the perpendicular and parallel elements of the polarizability tensor of the magnetite particle respectively, and $F(H, r, z)$ can be derived from Eq. (40) of Ref. 6, including the demagnetizing factor and the position dependence of the temperature in the Langevin function

$$
F(H, r, z)=\frac{1}{3}-\frac{16 \pi^{2}}{405} \frac{M^{2}\left\langle b^{3}\right\rangle}{\left(1+N_{d m} \chi\right)^{2} k_{B}^{2} T(r, z)^{2}} H^{2} .
$$

In the low field limit $\left(4 \pi\left\langle b^{3}\right\rangle M H\right) /\left[3 k_{B} T(1\right.$ $\left.\left.+N_{\mathrm{dm}} \chi\right)\right] \ll 1$, where $M$ and $(4 \pi / 3)\left\langle b^{3}\right\rangle$ are the magnetization and the average volume of the magnetic particles, respectively, $N_{\mathrm{dm}}$ is the demagnetization factor, and $\chi$ is the susceptibility of the sample. Note that the magneto-optical effect is coupled here to the thermal lens effect through the spatial temperature distribution and that the $z$ dependence (along the direction of the beam propagation) of the temperature has been introduced here by multiplying the second term in Eq. (1) with a factor $e^{-\alpha z}$ because the ferrofluid has a large absorption coefficient $\left(\alpha \sim 354 \mathrm{~cm}^{-1}\right)$.

Upon neglecting Fresnel variations in the phase ${ }^{4}$ when the thermal lens and magneto-optical effects are dominant,

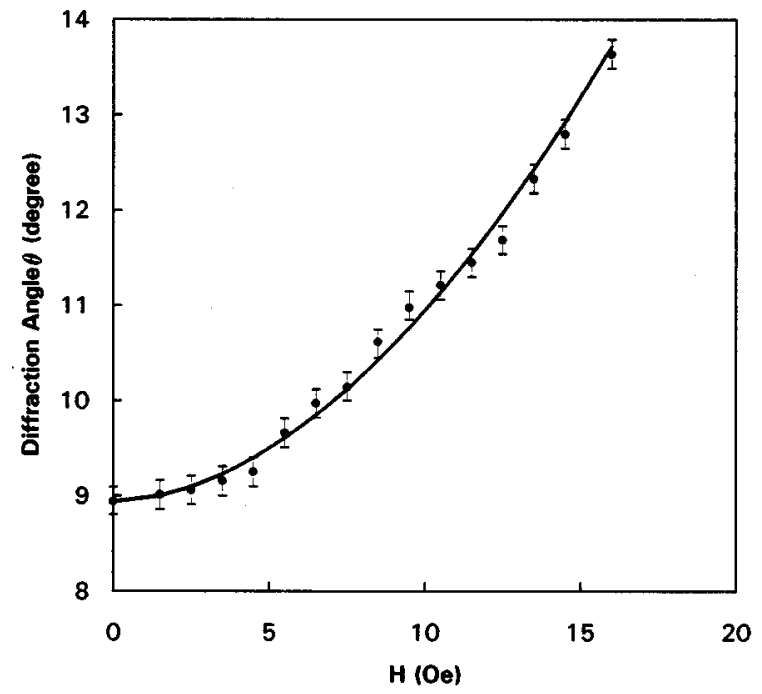

FIG. 3. The field dependence of the diffraction angle $\theta$ at low fields. Dots represent the experimental data, while the solid line is the least squares fit to $\theta=a+b H^{c}$, with $a=8.94, b=0.029$, and $c=1.84$.

the diffraction angle $\theta_{f}$ of the laser beam in the medium can be approximately given by ${ }^{4}$

$$
\theta_{f}=\left|\frac{\partial}{\partial r} \int_{0}^{L} \frac{\delta n(r, z)_{\mathrm{th}}+\delta n(r, z)_{H}}{m_{0}} d z\right|,
$$

where $m_{0}$ is the refractive index of the ferrofluid at room temperature and zero field. The combination of Eq. (4) with Eqs. (2), (3), and (1) gives the relationship between the diffraction angle $\theta$ and the applied magnetic field in the low field limit

$$
\theta_{f} \propto H^{2} .
$$

In Fig. 3, we plot the diffraction angle for the outermost ring on the screen as a function of applied fields. The solid line is the least-squares fit of our data to the function: $\theta=a+b H^{c}$, with $a=8.94, b=0.029$, and $c=1.84$, in reasonable agreement with Eq. (5). The angle $\theta$ is bigger than the diffraction angle $\theta_{f}$ in the ferrofluid because the refractive index of ferrofluid is greater than one. This correction should not alter the quadratic field dependence.

The angle resolution to the field is 0.5 Oe by using a visual inspection. We believe that this resolution can be further improved by a sensitive intensity detector. Therefore this magneto-optical effect can be used to develop a new type of optical sensor to probe magnetic fields as small as or smaller than 0.5 Oe.

Because the agglomeration of magnetic particles is expected in a field larger than a critical value, ${ }^{7}$ this technique can be also useful for detecting the onset of this aggregation from Eq. (3) for fields larger than the ones used in this work.

We thank Dr. R. E. Rosensweig for providing the ferrofluid sample used in this work. This work was partially supported by NSF Grant No. DMR-935478. 
${ }^{1}$ Yu. I. Dikanskii and A. O. Tsebers, Magnitnaya, Gidrodinamika No. 2, April-June, 47 (1990).

${ }^{2}$ J.-C. Bacri and D. Salin, J. Phys. Lett. 43, L771 (1982).

${ }^{3}$ W. Koechner, Solid State Laser Engineering (Springer, New York, 1988), p. 173.

${ }^{4}$ J. P. Gordon, R. C. C. Leite, R. S. Moore, S. P. S. Porto, and
J. R. Whinnery, J. Appl. Phys. 36, 3 (1965)

${ }^{5}$ F. W. Dabby, T. K. Gustafson, J. R. Whinnery, Y. Kohanzacleh, and P. L. Kelley, Appl. Phys. Lett. 16, 9, 362 (1970).

${ }^{6}$ J. J. M. Janssen and J. A. A. J. Perenboom, J. Magn. Magn. Mater. 81, 14 (1989).

${ }^{7}$ S. Yuan, T. Du, and W. Luo (unpublished). 